Revue des patrimoines

25 | 2014

Le patrimoine de la Grande Guerre

\title{
Le patrimoine postal de la Grande Guerre
}

\section{Grégory Aupiais}

\section{(2) OpenEdition}

\section{Journals}

Electronic version

URL: http://journals.openedition.org/insitu/11591

DOI: 10.4000/insitu. 11591

ISSN: 1630-7305

\section{Publisher}

Ministère de la Culture

\section{Electronic reference}

Grégory Aupiais, «Le patrimoine postal de la Grande Guerre », In Situ [Online], 25 | 2014, Online since 15 December 2014, connection on 25 June 2020. URL : http://journals.openedition.org/insitu/11591 DOI : https://doi.org/10.4000/insitu. 11591

This text was automatically generated on 25 June 2020.

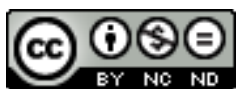

In Situ Revues des patrimoines est mis à disposition selon les termes de la licence Creative Commons Attribution - Pas d'Utilisation Commerciale - Pas de Modification 4.0 International. 


\title{
Le patrimoine postal de la Grande Guerre
}

\author{
Grégory Aupiais
}

1 Si la disparition de Jean Grelaud, l'un des trois ultimes rescapés de la Grande Guerre, survenue le 27 février 2007, avait suscité un certain émoi au sein des instances gouvernementales, celle de Louis de Cazenave, le 20 janvier 2008, sembla en revanche être passée plutôt inaperçue. Pourtant, c'est sans doute alors que l'option choisie par le président de la République le 18 novembre 2005, à l'occasion d'une réunion du Haut Conseil de la mémoire combattante de retenir «le principe d'obsèques solennelles de portée nationale pour le dernier poilu » avait désormais dépassé le stade de la fiction ${ }^{1}$. En effet, il devait désormais importer aux initiateurs institutionnels de ce projet, comme à leurs involontaires héritiers d'ailleurs, que derrière Lazare Ponticelli ne se cache pas un ultime survivant inconnu. Toutefois, le dernier de la « der des der » qui avait rejeté dès 2006 toute velléité cérémonielle, affirmant même que «ce serait un affront pour tous les autres ", a cependant très largement contribué à dédramatiser cette question ${ }^{2}$.

2 En effet, cet engagement au nom du peuple français reprenait en des termes plus mesurés une proposition de loi déposée le 15 novembre 2005 par la sénatrice UMP Bernadette Dupont ${ }^{3}$. Le caractère morbide de ce compte à rebours visant à désigner l'heureux et unique récipiendaire de l'hommage de la nation pouvait paraître singulièrement déplacé, tout $\mathrm{du}$ moins maladroit. Cette démarche s'inscrivait cependant dans la suite logique de la décision de Pierre Pasquini, alors ministre aux Anciens Combattants et Victimes de guerre, d'attribuer systématiquement la Légion d'honneur à tous ceux qui n'avaient pas encore été décorés. Elle en constitua même une sorte d'aboutissement. Les services de l'État entreprirent à partir du milieu des années 1990 un vaste recensement exhaustif avec l'aide du milieu associatif, abondamment relayé par la presse aussi bien locale que nationale.

3 Après des décennies de négation, d'oubli, puis d'indifférence, les anciens poilus ont brutalement accédé à un statut d'icônes mémorielles auxquels peu, sinon aucun d'entre eux, étaient véritablement préparés. La parole de ces hommes, désormais sacralisée, n’a 
pourtant plus grand chose à apporter d'un point de vue strictement scientifique. Les aspects événementiels, y compris les plus sombres, ne sont plus à explorer. Quant aux témoignages des combattants anonymes, ils abondaient déjà avant la fin du conflit. Quelques-uns, à l'image des carnets de guerre de Louis Barthas ou du recueil de lettres publié sous la direction de Jean-Pierre Guéno et d'Yves Laplume, ont même été postérieurement de véritables succès de librairie, profitant du contexte il est vrai favorable des cérémonies commémorant le $80^{\mathrm{e}}$ anniversaire de l'Armistice ${ }^{4}$.

Ainsi, il n'y a pas lieu de transposer à la Première Guerre mondiale l'affirmation d'Amadou Hampâté Bâ. Quand un poilu meurt, ce n'est pas en effet « une bibliothèque qui brûle $»^{5}$. En dépit de l'appel péremptoire lancé en 2003 par l'hebdomadaire ParisMatch, la sauvegarde des dernières voix du conflit ne revêt aucun caractère d'urgence. Toutefois, cette brutale hypertrophie, ou plutôt inflammation mémorielle, amène à s'interroger sur la nature et l'évolution des représentations de la Première Guerre mondiale, dont l'imagerie postale fut l'un des témoins privilégiés, bien que parmi les plus discrets.

\section{Du trauma à l'amnésie d'État}

De 1914 à 2013, la Première Guerre mondiale a constitué le thème central ou un simple arrière-plan pour 44 émissions de timbres-poste. Ces dernières ne représentent toutefois qu'un peu plus de $1 \%$ du nombre total de figurines émises en France métropolitaine depuis 1914, ce qui est relativement peu, car des thématiques touristiques, par exemple, en concentrent à elles seules une part beaucoup plus importante. Toutefois, la Grande Guerre fut indirectement à l'origine de deux innovations philatéliques majeures qui marquèrent durablement l'histoire postale française. C'est le 18 août 1914, quelques jours seulement après le déclenchement des hostilités, que la première figurine vendue avec une surtaxe au profit de la Croix-Rouge a été émise. Dans un premier temps, l'administration des Postes s'est contentée d'apposer sur un timbre-poste d'usage courant une surcharge composée d'une petite croix et mentionnant également le montant ajouté à la valeur faciale initiale. Un changement dans la continuité donc, mais l'expérience dut paraître concluante car elle fut renouvelée à peine un mois plus tard avec l'émission d'un timbre-poste spécifique. Sur le plan iconographique, cette innovation pouvait sembler bien modeste. Elle se matérialisait en effet par l'adjonction dans le coin gauche d'une figurine d'un petit pavé blanc très lisible reprenant l'ensemble des informations contenues dans la précédente surcharge, à savoir une petite croix non plus rouge mais orange et le montant de la surtaxe (fig. 1).

Figure 1

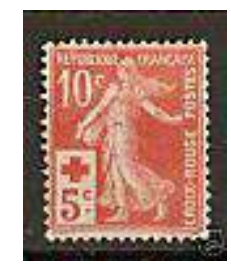

Semeuse camée avec une surtaxe au profit de la Croix-Rouge, 1914, 10 c. + 5 c., rouge. Dessin d'après Louis Oscar Roty, gravure de Jean-Baptiste Lhomme.

(c) http://www.phil-ouest.com/. 
Le succès rencontré par ces deux premières émissions invitait sans doute à voir plus grand encore. Ainsi à partir d'août 1917, une série de 7 timbres-poste sur le thème générique de la Première Guerre mondiale fut mise en circulation. Pour en assurer le succès commercial, et donc caritatif, l'administration des Postes décida de rompre avec la symbolique monétaire utilisée jusqu'alors d'une manière exclusive par l'imagerie postale. En dépit d'un format réduit, le timbre-poste devenait un nouvel espace d'expression iconographique, encore très largement à créer. En sept "clichés » philatéliques furent ainsi évoquées tour à tour les conséquences humaines et sociales du conflit, sans oublier la grandeur d'une nation et de la résistance française. Les deux allégories ne firent pas preuve d'originalité. Elles magnifièrent le sentiment national à travers la frise de François Rude représentant la Marseillaise, située sur l'un des piédroits de l'Arc de Triomphe, et la réplique belfortaine du lion de la place DenfertRochereau. L'unique représentation réelle de la Grande Guerre est un paysage de tranchées aussi lunaire qu'aseptisé, déserté par les combattants des deux camps (fig. 2)

Figure 2

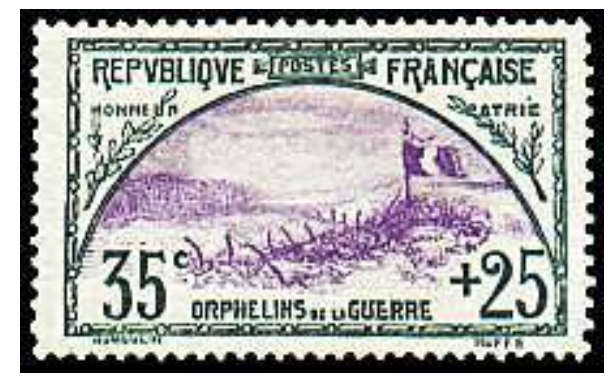

Tranchée et drapeau, 1917, 35 c. + 25 c., ardoise et violet. Dessin de Louis Dumoulin, gravure de Léon Ruffé.

(c) http://www.phil-ouest.com/.

7 Cette vision idyllique, bien qu'un peu oppressante, se retrouve quand sont évoqués les blessés de guerre dont la réalité sociale était plus proche du tableau de Jean GaltierBoissière que de ce cadre bucolique peuplé de religieuses infirmières aussi attentives que dévouées ${ }^{6}$ (fig. 3). La délicate thématique des veuves et orphelins de guerre bénéficia d'une approche graphique beaucoup moins édulcorée. En effet, si les réalités $\mathrm{du}$ front échappaient pour une large part à l'opinion publique, certaines de ses conséquences collatérales la touchaient de plein fouet et l'image de la veuve et de ses stigmates vestimentaires fit son entrée dans l'univers visuel ordinaire des Français. Rétrospectivement, la vision de cette femme éplorée, enveloppée dans un large voile noir, errant dans un cimetière, peut paraître sinistre. Toutefois ces silhouettes fugaces faisaient partie intégrante de la vie quotidienne durant le premier conflit mondial, au cours duquel périrent près de 1,4 millions de soldats (fig. 4). De plus, cette première impression morbide fut tempérée par les autres figurines relatives aux orphelins de guerre et au travail des femmes, car dans l'un et l'autre cas, que ce soit à travers le regard de ces deux enfants tournés vers l'horizon ou le thème agrarien de la femme au labour, c'est la capacité de la nation à se régénérer qui se trouvait ainsi magnifiée. 


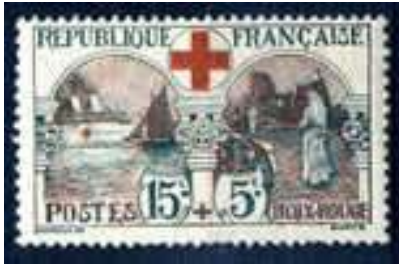

Infirmière, 1918, 15 c. + 5 c., noir et rouge. Dessin de Louis Dumoulin, gravure de Léon Ruffé.

(c) http://www.phil-ouest.com/.

Figure 4

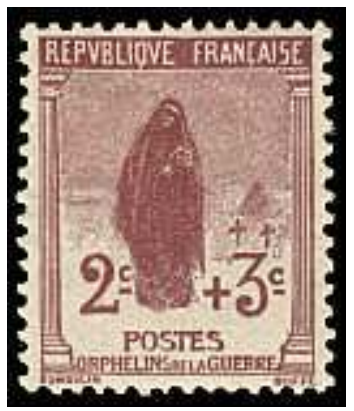

Veuve au cimetière, 1917, 2 c. + 3 c., brun-lilas. Dessin de Louis Dumoulin, gravure de Léon Ruffé. (c) http://www.phil-ouest.com/.

Dès sa création le $1^{\mathrm{er}}$ janvier 1849 , l'imagerie postale avait montré une certaine perméabilité à l'évolution de la conjoncture politique. Ainsi, le profil de Cérès, d'abord choisi, avait été remplacé par celui de Napoléon III, tête nue dans un premier temps, quand ce dernier ne s'appelait encore que Charles Louis Napoléon Bonaparte, puis couronné de lauriers après le 2 décembre 1852 . Une dynamique qui ne fut nullement contrariée par la défaite de 1870 . Toutefois, cette aptitude osmotique se limitait strictement au domaine politique et aucune possibilité d'extension au temps présent et à son histoire très contemporaine n'était alors envisagée. Cette première série de 7 timbres-poste marque ainsi symboliquement l'entrée de l'événement dans ce corpus iconographique d'un genre nouveau. Pour expérimentale qu'elle soit, elle dut cependant rencontrer un accueil favorable car elle fut de nouveau mise en circulation en 1922, puis en 1926. D'abord avec une nouvelle surtaxe unique de 25 centimes, alors que pour la première émission son montant était variable, allant jusqu'à doubler la valeur faciale. Cette logique fut reprise en 1926 avec toutefois une nette diminution de l'ensemble des plus-values caritatives. Ce hoquet philatélique, dont l'administration des Postes fut assez coutumière, du moins dans la première moitié $\mathrm{du} \mathrm{xx}^{\mathrm{e}}$ siècle, sonna d'ailleurs le glas de l'ensemble des représentations du conflit qui disparurent du paysage philatélique pendant près d'une décennie. La réapparition de cette thématique en 1936 ne fut pas, dans un premier temps du moins, synonyme de renouveau. Elle resta cantonnée dans un registre morbide, dont elle semblait d'ailleurs avoir du mal à se défaire. En effet, cette paire de figurines représentait le monument aux morts canadiens édifié à Vimy. C'est sans doute la montée des tensions sur la scène internationale qui imposa une approche renouvelée de la mémoire postale de la Grande Guerre. Le misérabilisme sinistre de l'immédiat après-guerre s'effaça devant l'affirmation d'une force tranquille avant la lettre. Une autre paire émise en 1938 à la 
gloire de l'infanterie française évoque par son arrière-plan le passé militaire récent. Mais il n'y a nulle trace de désespoir dans la placide détermination de cette sentinelle. La paix retrouvée depuis 20 ans semblait en effet de plus en plus menacée sous les coups de boutoir de la future coalition des puissances de l'Axe, mais un espoir ténu de la préserver subsistait encore. C'est donc dans un contexte diplomatique bien particulier que se déroulèrent les différentes manifestations organisées à l'occasion du 11 novembre sur l'ensemble du territoire national et dont le timbre-poste constituait alors l'un des éléments incontournables. De cette démonstration de force martiale ne devait cependant transparaître aucune forme d'agressivité belliqueuse. Aussi, aux véhicules militaires et aux soldats en armes furent préférés des porte-drapeaux symbolisant la solidité des alliances et indirectement, le caractère vain de tout esprit de revanche de la part des anciens agresseurs (fig. 5).

Figure 5

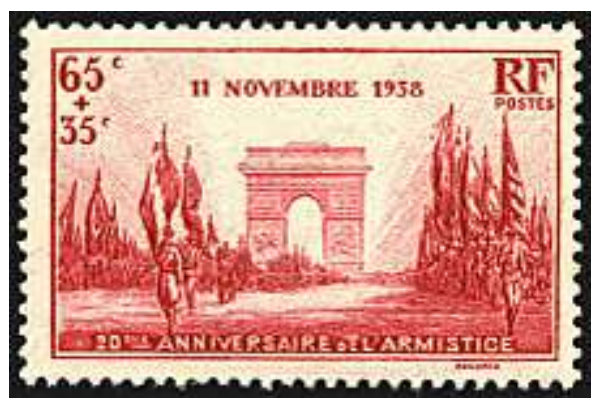

$20^{\circledR}$ anniversaire de l'Armistice, 1938, 65 c. + 35 c., rouge carminé. Dessin et gravure de Georges Léo Degorce.

(C) http://www.phil-ouest.com/.

Cette marche vers l'inéluctable ne devait pourtant connaître aucun répit. Néanmoins, l'administration des Postes ne s'y associa que très tardivement. En effet, le 23 juin 1939 fut émis un timbre-poste sur Verdun dans le cadre d'une série générale sur les sites et monuments remarquables. Toutefois, aussi étonnant que cela puisse paraître, ce fut l'aspect touristique qui prima avec une représentation de la porte chaussée dont l'évocation semblait toutefois bien éloignée de l'événement, si ce n'est par ce discret rappel à travers une date, "1916», et dans le coin gauche, un casque posé sur une branche de lauriers. Quelques mois auparavant elle avait de nouveau sombré dans le pathos avec une figurine émise à la mémoire des victimes civiles de la Grande Guerre. Ce n'est que tardivement, à partir du mois de mai 1940, qu'un véritable appel aux lares du glorieux passé militaire récent fut lancé. Les effigies des maréchaux Joseph Joffre, Ferdinand Foch et Joseph Simon Gallieni, le conquérant de Madagascar, mais aussi l'un des artisans de la victoire de la Marne, firent ainsi leur apparition dans l'imagerie postale française. Ils furent rejoints un peu tardivement, le 12 octobre 1940, sur cet autel républicain improvisé par l'aviateur Georges Guynemer dont le souvenir militaire héroïque, presque chevaleresque, était encore très présent, tant du côté français qu'allemand. En 1941, le secrétariat d'État à l'aviation avait demandé, et obtenu, l'émission d'une vignette postale avec une surtaxe au profit de ses œuvres sociales. Pour gagner du temps et satisfaire ce commanditaire qui souhaitait la mettre en vente parallèlement à une semaine de sensibilisation, le secrétaire général des PTT, Vincent Di Pace, proposa de réutiliser avec une surcharge le timbre-poste à l'effigie de Georges Guynemer dont il restait des stocks importants, voire d'effectuer un autre tirage avec 
une nouvelle couleur. Il renonça cependant très rapidement à ce projet car un receveur parisien avait rapporté à ses services que des officiers allemands philatélistes, effectuant des achats dans un bureau de poste, avaient demandé : « Tous les timbres en cours à l'exception de Guynemer ». Il fit part de cette anecdote au secrétaire d'État aux communications qui, après avoir consulté l'amiral Darlan, jugea aussi plus prudent de réaliser un autre timbre-poste en dépit de l'investissement non négligeable que représentait la conception d'un nouveau poinçon'.

\section{D'une mémoire l'autre ${ }^{8}$}

10 Les quelques mois précédant l'entrée en guerre de la France furent cependant le modeste point d'orgue de la diffusion des représentations de la Première Guerre mondiale dans l'imagerie postale. Ce dernier apparaît rétrospectivement d'autant plus remarquable que le régime de Vichy fut en parallèle synonyme de cinquante mois d'absence et d'oubli. Cette parenthèse constitue même un véritable paradoxe politique. En effet, la Grande Guerre était le socle de la popularité du nouveau chef de l'État qui entendait fonder la reconstruction du pays sur le corps des anciens combattants fédéré par la loi du 29 août 1940 en une association unique, la Légion française des combattants. Pourtant, les autorités vichyssoises essayèrent d'effacer jusqu'à la mémoire de l'événement. Des mesures d'interdiction de l'ensemble des manifestations commémoratives habituellement organisées dans le cadre du 11 Novembre furent édictées. L'initiative en revenait certes à l'occupant. Mais le gouvernement de Vichy veilla jusqu'à la Libération à la plus stricte application de ces dispositions. Il accepta même de se compromettre dès novembre 1940 en publiant une note officielle dans les journaux. La portée politique de cette décision, presque conjointe, allait bien au-delà de la simple, et par ailleurs compréhensible, délicatesse diplomatique car derrière le symbole, c'est l'esquisse d'une stratégie gouvernementale à plus long terme qui semblait se dessiner. Mais l'imagerie postale, comme la nature, a horreur du vide et en règle générale un espace ainsi laissé vacant est rapidement comblé. Quelques mois suffisent même pour la rendre totalement amnésique et il est rare qu'elle consente ensuite à réhabiliter ce qu'elle a un temps voué au néant. Dès le 15 novembre 1944, le passé immédiat s'effaça devant le temps présent et à la Première Guerre mondiale succéda la seconde. Un coq gaulois et une croix de Lorraine, ainsi qu'un profil de Marianne encadré de lauriers, constituèrent les prémices d'un véritable raz de marée iconographique. Au fil des mois même cette présence thématique s'affirma, au point de devenir omnipotente. En effet, en 1945, seule une minorité de timbres-poste ne comportèrent aucune allusion à la Seconde Guerre mondiale. Ce caractère prégnant semble s'estomper dès l'année suivante. Toutefois, si les représentations directes du conflit, ou de ses conséquences les plus visibles, tendent effectivement à reculer, ce dernier transparaitt toujours en filigrane à travers le portrait de certains personnages devenus emblématiques. Quand l'arrière-plan n'était pas encore plus explicite. La diffusion de ce corpus thématique original disposait ainsi des bases nécessaires pour s'installer dans la longue durée. En outre, cette personnalisation lui permettait de sortir du cadre étroit du calendrier commémoratif. Ces apparitions " anarchiques », car adaptées à chaque individu célébré, contribuèrent à en renforcer la présence et ce, d'autant plus que la plupart des faits d'armes de la France combattante furent en parallèle mis en exergue, y compris le débarquement avorté de Narvik. Ils donnèrent même lieu en 1969 à l'émission d'une série presque exhaustive de 6 figurines. Entre ces 
évocations martiales et la résistance intérieure, l'espace laissé à l'expression d'autres mémoires contemporaines s'en trouvait singulièrement réduit. Aucun timbre-poste ne vint marquer en 1948 le trentième anniversaire de l'Armistice. La décennie suivante sembla commencer sous des auspices plus favorables avec en 1951 un premier timbreposte à l'effigie de Clemenceau. Bien que la mise en vente de ce dernier soit intervenue un 11 novembre, le contexte historique, généralement matérialisé par l'arrière-plan, voire la légende, a totalement été éludé, ou presque. Il ne transparait timidement qu’à travers quelques barbelés en ombres chinoises et représentant dans le lointain d'une manière aussi symbolique que discrète un paysage de tranchées. Cet exemple demeura cependant aussi isolé que représentatif car les rares personnages comme Louis Pergaud ou Henri Alain-Fournier, qui auraient pu constituer comme autant d'allusions à la Première Guerre mondiale, se virent appliquer un traitement graphique aussi synonyme d'amnésie. Ainsi, dans l'imagerie postale, la Grande Guerre ne peut prétendre dès la seconde moitié $\mathrm{du} x \mathrm{x}^{\mathrm{e}}$ siècle qu'à une mémoire minimale s'inscrivant dans un cycle décennal. À défaut de pouvoir prétendre à l'exhaustivité, ce corpus iconographique eut au moins le mérite de la concision, illustrant décennie après décennie l'évolution des représentations du conflit au sein de l'opinion publique. Les années 50 restèrent ainsi marquées par le martyrologue hérité de l'immédiat aprèsguerre. Mais le temps avait passé, et sur la terre meuble de ces tombes anonymes, une végétation touffue avait poussé, aussi luxuriante qu'annonciatrice d'oubli (fig. 6). La disparition progressive de cette dimension morbide n'était toutefois que l'étape préparatoire à l'idéalisation du souvenir, car en 1968, ce réalisme aseptisé s'effaça au profit d'une approche allégorique renouvelée qui n'était désormais plus empruntée à d'autres champs chronologiques. De l'horreur des tranchées, il ne subsistait plus que cette vision évanescente d'une victoire ailée bleu horizon tenant dans chacune de ses mains une couronne de lauriers tressés. Nimbée d'une aura surnaturelle, elle flottait au-dessus d'un Arc de Triomphe à la base duquel jaillissait, à une hauteur plutôt inhabituelle, la flamme de la tombe du soldat inconnu (fig. 7).

Figure 6

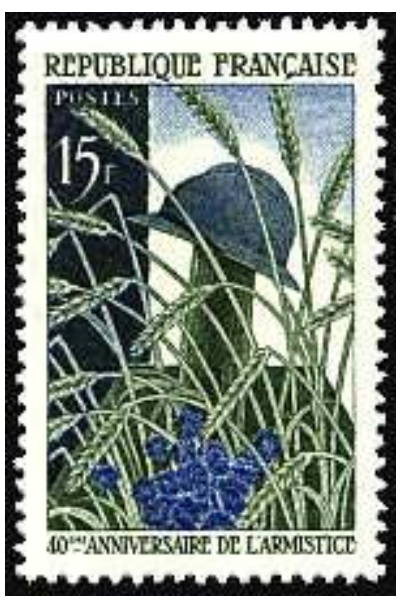

40 anniversaire de l'Armistice, 1958, 15 f., vert et bleu. Dessin et gravure de Pierre Gandon.

(c) http://www.phil-ouest.com/. 
Figure 7

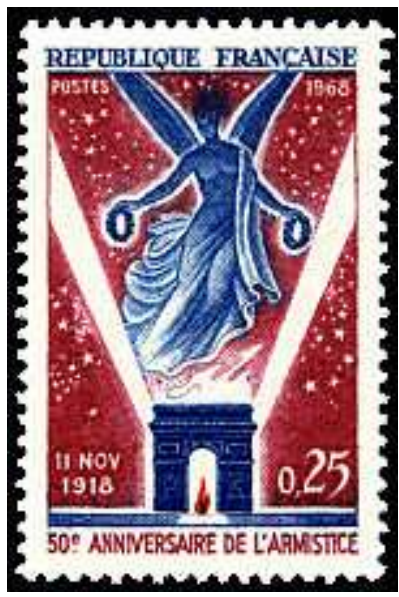

$50^{\mathrm{e}}$ anniversaire de l'Armistice, 1968, 25 c., carmin et bleu. Dessin et gravure de Pierre Gandon.

(c) http://www.phil-ouest.com/.

11 Une symbolique assez analogue fut d'ailleurs utilisée pour commémorer le quarantième anniversaire de l'Armistice sur le front d'Orient, première allusion au caractère mondial du conflit. À un détail près cependant, car la Tour blanche de Salonique se substitua à la désormais classique perspective des Champs Élysées. La décennie suivante, coïncidant avec le cinquantième anniversaire de l'Armistice, sembla marquer la fin du temps présent. La figurine émise à cette occasion comportait en effet pour la première fois une dimension presque patrimoniale avec une représentation du wagon de Rethondes, tout du moins de sa réplique, l'original emporté en Allemagne après la signature de l'armistice le 22 juin 1940 ayant été incendié lors d'un bombardement en avril 1945. Après cette première tentative expérimentale, que n'auraient pas reniée les tenants de l'histoire bataille, ce fut au tour des combattants de sortir de l'ombre. D'abord en 1986, à travers une allégorie souffrante puis en 1988, ils semblèrent émerger des brumes de la mémoire collective. Une impression suggérée d'ailleurs par la création originale de Michel Durand-Mégret et Pierre Albuisson, qui les représentait telles des ombres évanescentes (fig. 8). Le caractère tardif de cette découverte a de quoi surprendre. En effet, les témoignages, qu'ils soient littéraires, institutionnels ou individuels abondaient avant même la fin du conflit. Mais la société française dans son ensemble a mis beaucoup plus longtemps avant de pouvoir les accepter, voire simplement de les entendre, et c'est en définitive plutôt récemment que beaucoup des derniers acteurs involontaires de cette tragédie retrouvèrent parallèlement le courage de transmettre aux générations futures cette part d'eux-mêmes qui ne les avait jamais quittés9. Cette initiative resta cependant isolée car en 1998, à ces évocations " humanistes " fut préférée une composition des plus classiques élaborée à partir de fragments de drapeaux brisés et savamment entremêlés. 
Figure 8

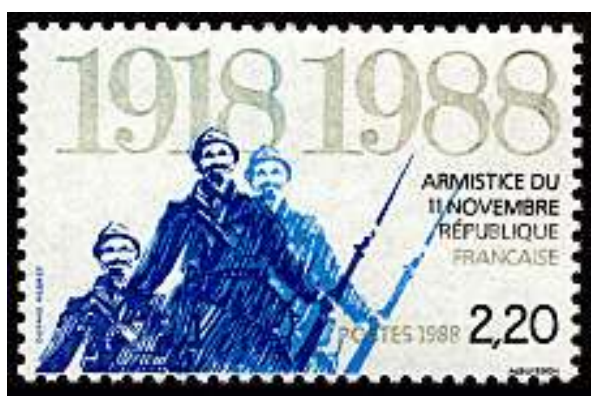

70 anniversaire de l'Armistice, 1988, 2 f. 20 c., bleu et noir. Dessin de Michel Durand-Mégret, gravure de Pierre Albuisson.

(c) http://www.phil-ouest.com/.

Le sort qui sera réservé à l'imagerie postale de la Première Guerre mondiale au cours du $\mathrm{XXI}^{\mathrm{e}}$ siècle reste ainsi encore très largement à définir. Avec un premier timbre-poste émis en 2006, il semble déjà que ce cycle décennal pour ainsi dire immuable soit quelque peu contesté. En outre, le choix de l'Ossuaire de Douaumont indique également une volonté réaffirmée de mettre en exergue une dimension souffrante, bien éloignée de certaines allégories nationales qui avaient vanté jusqu'à l'écœurement l'aspect sacrificiel (fig. 9). L'année 2008 marquant le quatre-vingt-dixième anniversaire de l'Armistice semble être allée dans ce sens, car le timbre-poste émis à cette occasion célébra la fin des combats et non la victoire à proprement parler (fig. 10). C'est à l'année du centenaire qu'il reviendra cependant de confirmer ou non ce qui peut s'apparenter à l'esquisse d'une tendance, car il est prévu dans l'article 2 de l'arrêté du 4 octobre 2012 fixant le programme philatélique une série commémorative relative au centenaire de la Première Guerre mondiale ${ }^{10}$. Il est probable même qu'elle posera quelques-unes des bases essentielles des représentations du conflit pour les années à venir, d'autant que leur pérennité paraît singulièrement menacée. En effet, des voix demandant la suppression du 11 Novembre comme jour chômé se sont élevées récemment. Les arguments invoqués s'interrogent sur l'opportunité de maintenir une telle commémoration dans le contexte politique européen, alors qu'il ne reste pratiquement plus de rescapés à honorer d'une ultime décoration. Toutefois, limiter ainsi la portée symbolique réelle aux seuls anciens combattants paraît plutôt réducteur, tout particulièrement dans ce contexte global d'hypertrophie mémorielle commun aux sociétés occidentales contemporaines. Au demeurant, cette proposition masque mal, derrière une générosité de façade, des arrière-pensées commerciales à court terme faisant peu de cas tant de la vie humaine que de la détresse morale et sociales de ces familles qui mirent bien souvent près de deux générations à effacer les stigmates du conflit. Un jour chômé ne conditionne certes pas le souvenir. Mais il donne l'opportunité, en arrêtant le cours ordinaire de la vie, et pour qui le souhaite, de s'interroger sur le passé afin d'envisager sur quelles bases il est possible de construire l'avenir. 
Figure 9

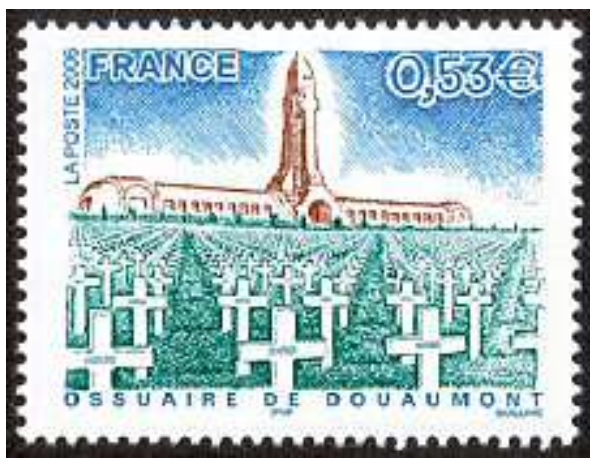

Ossuaire de Douaumont, 2006, 0,53 €, multicolore. Dessin et gravure de René Quivillic.

(c) http://www.phil-ouest.com/.

Figure 10

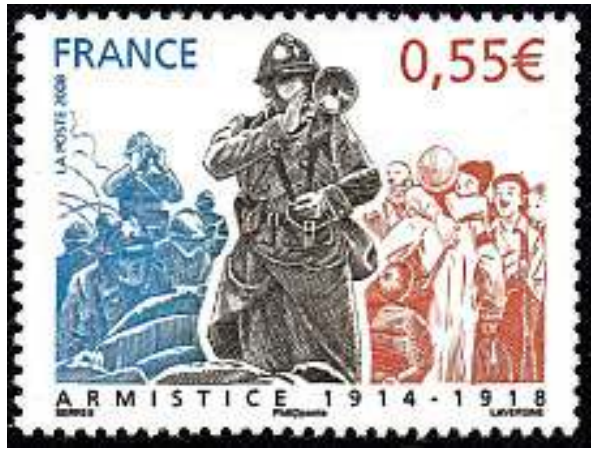

$90^{\mathrm{e}}$ anniversaire de l'Armistice, 2008, 0,55€, bleu, gris et rouge. Dessin de Patrice Serres, gravure d'André Lavergne.

(c) http://www.phil-ouest.com/.

\section{NOTES}

1. - OFFENSTADT, Nicolas. «Le pays a un héros: le dernier poilu ». L'Histoire (230), mai 2007, p. 25-26. «Le Haut Conseil de la mémoire combattante est chargé d'éclairer le Président de la République sur toutes les questions relatives au devoir de reconnaissance par la nation de la sauvegarde de la mémoire des guerres ou des conflits contemporains et de la préservation des valeurs du monde combattant ». Décret n 97-11 du 9 janvier 1997, Journal officiel, 11 janvier 1997 (9), p. 464.

2. - L'Express, 24 janvier 2008 (2951), p. 82-86.

3. - La sénatrice Bernadette Dupont proposait en effet initialement l'organisation de funérailles nationales. Voir le site : http://www.senat.fr/leg/ppl05-080.html.

4. - BARTHAS, Louis. Les carnets de guerre de Louis Barthas, tonnelier, 1914-1918. Paris : La Découverte, 1997 ; GUENO, Jean-Pierre et LAPLUME, Yves (dir.). Paroles de Poilus. Lettres et carnets du front, (1914-1918). Paris : Librio, 1998. 
5. - Formule prononcée en 1962 à la tribune de l'Unesco.

6. - GALTIER-BOISSIERE, Jean. « Défilé des mutilés. 14 juillet 1919 ».

7. - AUPIAIS, Grégory. Outils de propagande ou miroirs de l'opinion publique : les émissions de timbresposte en France métropolitaine de 1940 à 1944. Mémoire de DEA, École des hautes études en sciences sociales, 2000, p. 59.

8. - Un évident parallèle chronologique rendait particulièrement opportune l'utilisation de cet effet de style popularisé par Céline dans le titre D'un château l'autre. La suppression du "à » permettant de traduire du point de vue littéraire le mouvement et la rapidité. ALLIOT, David. Louis-Ferdinand Céline. Paris : Éditions Horay (coll. « En Verve »), 2004, p. 11.

9. - Frédéric Mathieu rapporte en effet que Jean Grelaud, décédé le 27 février 2007, n'a accordé sa première interview qu'en novembre 2006. Voir le site: http://derdesder.free.fr/bio_veteran/ grelaud.html.

10. - Arrêté du 4 octobre 2012 fixant le programme philatélique de l'année 2013 (compléments) et de l'année 2014, $1^{\mathrm{e}}$ partie. Voir le site: http://www.legifrance.gouv.fr/affichTexte.do? cidTexte=LEGITEXT000027010904.

\section{ABSTRACTS}

The histories of the First World War and of postal services have long since been intertwined with close and complex connections. The letters sent by soldiers to their families and their loved ones today represent one of the principal sources for the memory of the war. But these letters are no more than a myriad of individual stories which do not allow for even the vaguest of collective representations. Generally associated with these letters, postage stamps also offer a reflection of the story of the war. The stamp is a source which is both revealing and poorly appreciated, paid little attention on account of its tiny size and the long-term familiarity of its appearance.

La Première Guerre mondiale et l'histoire postale entretiennent depuis longtemps des rapports étroits et complexes. Les lettres adressées par les combattants à leur famille ou à leurs proches sont aujourd'hui l'une des sources privilégiées de la mémoire du conflit. Il ne s'agit pourtant que d'une myriade de témoignages individuels dont la collation ne permet pas d'appréhender, même de manière approximative, les représentations collectives. Généralement associés à ces correspondances, les timbres-poste en constituent en revanche un reflet, aussi révélateur qu'ignoré, car très largement desservi tant par la modestie du format que par une paradoxale pérennité.

\section{INDEX}

Mots-clés: timbres-poste, histoire postale, Première Guerre mondiale, politiques symboliques

Keywords: stamps, postal history, First World War, symbolic politics 
AUTHOR

GRÉGORY AUPIAIS

Ingénieur d'études, université Paris Diderot - Paris 7 gregory.aupiais@univ-paris-diderot.fr 